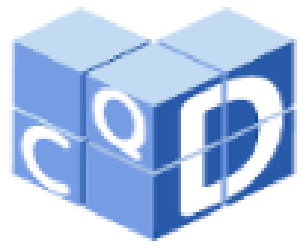

Revista Eletrônica

Paulista de Matemática

ISSN 2316-9664

Volume 18, jul. 2020

Francisco Evamar Barros Instituto Federal de Educação, Ciências e Tecnologia do Estado do Ceará (IFCE)

fco.evamar@gmail.com

Francisco Régis Vieira Alves Instituto Federal de Educação, Ciências e Tecnologia do Estado do Ceará (IFCE)

fregis@ifce.edu.br

\section{Funções geradoras dos números poligonais e dos números hipertetraédricos $\boldsymbol{k}$-dimensionais}

Generating functions of polygonal numbers and k-dimensional hypertetrahedral numbers

\begin{abstract}
Resumo
No presente artigo, discutimos sobre as funções geradoras de duas classes de sequências dos números figurados que contém os números triangulares, a primeira é referente a classe dos números poligonais que é uma extensão m-gonal dos números triangulares e a segunda, referente aos números simplexos, também conhecidos como números hipertetraédricos $k$-dimensionais, que se refere aos análogos dos números triangulares em dimensões arbitrárias. Este trabalho apresenta uma generalização de funções geradoras bem como as recorrências lineares e homogêneas das sequências estudadas por meio de procedimentos algébricos, com a possibilidade de aplicações em qualquer outra sequência seja ela de um números figurado ou não que possua uma recorrência do tipo linear e homogênea, por fim, apresenta uma solução de funções geradoras para os números simplexos por meio de operações envolvendo funções geradoras de dimensões inferiores, partindo sempre da sequência dos números triangulares.
\end{abstract}

Palavras-chave: Números Poligonais. Números Hipertetraédricos. Números figurados.

\begin{abstract}
In the present paper, we have discussed the generating functions by two sequence classes of the figurate numbers containing the triangular numbers, the first is the polygonal numbers class which is an extension $\mathrm{m}$-gonal triangular numbers and the second, referring to the simplex numbers, also known as k-dimensional hypertetrahedral numbers, which refers to analogues of triangular numbers in arbitrary dimensions. This work presents a generalization of generating functions as well the linear and homogeneous recurrences of the sequences studied by algebraic procedures, with the possibility of applications in any other sequence, whether it is a figurate numbers with or without a recurrence of the sequence. Finally, present a solution of generating functions for simplex numbers by operations involving generating functions of smaller dimensions, always starting from the sequence of triangular numbers.

Keywords: Polygonal Numbers. Hypertetrahedral Numbers. Figurate Numbers.
\end{abstract}




\section{Introdução}

As funções geradoras são ferramentas valiosas usadas para resolver vários problemas principalmente em análise combinatória, porém também podem ser úteis em outros campos da matemática como no estudo de sequências. O estudo destas funções permite encontrar a solução de recorrências lineares com coeficientes constantes, Koshy (2001); Vieira e Alves (2019), como a sequência de Fibonacci, Lucas e Pell, inclusive das potências destas sequências, Mansour (2004).

Para determinar a função geradora de uma sequência infinita de inteiros segundo Deza, E. e Deza, M. M. (2012), tomaremos dois polinômios.

Seja $f(x)=a_{0}+a_{1} x+a_{2} x^{2}+\cdots+a_{m} x^{m}$ e $g(x)=b_{0}+b_{1} x+b_{2} x^{2}+\cdots+b_{n} x^{n}$, com $m<n$, de modo que o quociente $\frac{f(x)}{g(x)}$, tenha como resultado a função $c_{0}+c_{1} x+c_{2} x^{2}+\cdots+c_{m} x^{m}+\cdots$ $+c_{n} x^{n}+\cdots$, esta função é chamada de geradora da sequência $c_{0}, c_{1}, c_{2}, \ldots, c_{m}, \ldots, c_{n}, \ldots$, sendo assim, $f(x)=g(x) \cdot\left(c_{0}+c_{1} x+c_{2} x^{2}+\cdots+c_{m} x^{m}+\cdots+c_{n} x^{n}+\cdots\right)$, que podemos reescrever como $a_{0}+a_{1} x+a_{2} x^{2}+\cdots+a_{m} x^{m}=\left(b_{0}+b_{1} x+b_{2} x^{2}+\cdots+b_{n} x^{n}\right) \cdot\left(c_{0}+c_{1} x+c_{2} x^{2}+\cdots+c_{m} x^{m}+\cdots+c_{n} x^{n}+\cdots\right)$. Então podemos determinar o valor para cada $a_{i}, \operatorname{com} i \geq \mathbb{N}$. Com base na igualdade, temos que:

$a_{0}=b_{0} c_{0}$

$a_{1}=b_{0} c_{1}+b_{1} c_{0}$

$a_{2}=b_{0} c_{2}+b_{1} c_{1}+b_{2} c_{0}$

$a_{3}=b_{0} c_{3}+b_{1} c_{2}+b_{2} c_{1}+b_{3} c_{0}$

$\cdots$

$a_{m}=b_{0} c_{m}+b_{1} c_{m-1}+b_{2} c_{m-2}+\cdots+b_{m} c_{0}$

Ainda temos que os valores de $b$ e $c$ maiores que $m$, são todos nulos ou seja:

$0=b_{0} c_{m+1}+b_{1} c_{m}+b_{2} c_{m-1}+\cdots+b_{m+1} c_{0}$

$0=b_{0} c_{n}+b_{1} c_{n-1}+b_{2} c_{n-2}+\cdots+b_{n} c_{0}$

$0=b_{0} c_{n+1}+b_{1} c_{n}+b_{2} c_{n-2}+\cdots+b_{n+1} c_{0}$

$0=b_{0} c_{n+k}+b_{1} c_{n+k-1}+b_{2} c_{n+k-2}+\cdots+b_{n+k} c_{0}$

Agora vejamos que dada uma sequência qualquer, podemos determinar uma recorrência linear de modo que essa sequência seja a solução da recorrência, então tomando como base a sequência $c_{0}, c_{1}, c_{2}, \ldots, c_{m}, \ldots, c_{n}, \ldots$, e a recorrência linear $b_{0} c_{n+k}+b_{1} c_{n+k-1}+b_{2} c_{n+k-2}+\cdots+b_{n+k} c_{0}$, sendo $b_{0}, b_{1}, b_{2}, b_{3}, \ldots, b_{n}$ os coeficientes dessa recorrência, assim, podemos determinar os valores de $a$ para o maior índice $m$ possível em relação a $n$ ou seja, $n-1$, usando os valores inicias da recorrência linear, então podemos reescrever $a_{0}+a_{1} x+a_{2} x^{2}+\cdots+a_{m} x^{m}=\left(b_{0}+b_{1} x+b_{2} x^{2}+\right.$ $\left.\cdots+b_{n} x^{n}\right)\left(c_{0}+c_{1} x+c_{2} x^{2}+\cdots+c_{m} x^{m}+\cdots+c_{n} x^{n}+\cdots\right)$ com índices $n$. Desse modo, temos que: $a_{0}+a_{1} x+a_{2} x^{2}+\cdots+a_{n-1} x^{n-1}=\left(b_{0}+b_{1} x+b_{2} x^{2}+\cdots+b_{n} x^{n}\right)\left(c_{0}+c_{1} x+c_{2} x^{2}+\cdots+c_{n} x^{n}\right)$ ou ainda 
$\frac{a_{0}+a_{1} x+a_{2} x^{2}+\cdots+a_{n-1} x^{n-1}}{b_{0}+b_{1} x+b_{2} x^{2}+\cdots+b_{n} x^{n}}=c_{0}+c_{1} x+c_{2} x^{2}+\cdots+c_{n} x^{n}$ e, definindo os valores para $a$ com base no índice $n$.

$a_{0}=b_{0} c_{0}$

$a_{1}=b_{0} c_{1}+b_{1} c_{0}$

$a_{2}=b_{0} c_{2}+b_{1} c_{1}+b_{2} c_{0}$

$a_{3}=b_{0} c_{3}+b_{1} c_{2}+b_{2} c_{1}+b_{3} c_{0}$

$\cdots$

$a_{n-1}=b_{0} c_{n-1}+b_{1} c_{n-2}+b_{2} c_{n-3}+\cdots+b_{n-1} c_{0}$

Sendo assim, utilizaremos nas seções subsequentes, o modelo da função geradora genérica $\frac{a_{0}+a_{1} x+a_{2} x^{2}+\cdots+a_{n-1} x^{n-1}}{b_{0}+b_{1} x+b_{2} x^{2}+\cdots+b_{n} x^{n}}=c_{0}+c_{1} x+c_{2} x^{2}+\cdots+c_{n} x^{n}$, para determinarmos as funções que geram cada sequência apresentada, buscando regularidades que nos forneçam argumentos de generalização das famílias de sequências que serão apresentadas.

\section{A função geradora dos números poligonais}

Buscaremos agora determinar as funções geradoras dos números poligonais, para isso, tudo que precisamos é de uma sequência, sua recorrência e seus valores iniciais e, empregando elementos aritméticos, podemos encontrar sua função geradora, vamos tomar como exemplo a sequência dos números triangulares $(1,3,6,10,15, \ldots)$.

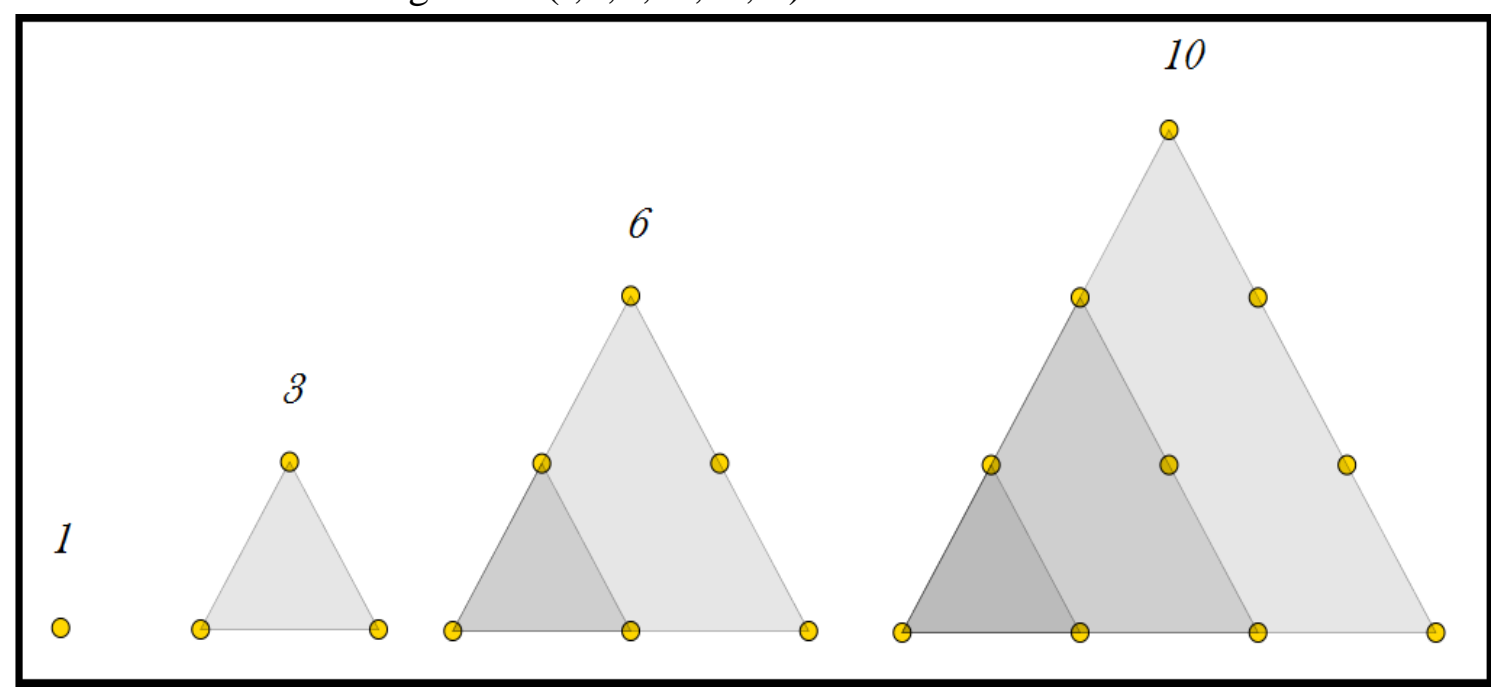

Figura 1 - Os quatro primeiros números triangulares.

Fonte: elaboração dos autores

A fórmula para se encontrar o próximo número triangular a partir do anterior é: $S_{3}(n+1)=S_{3}(n)+(n+1)$, (ALVES; BORGES NETO; MAIA, 2012), que é uma recorrência linear não homogênea e para encontrar a recorrência linear homogênea relacionada a essa sequência, teremos que usar alguns processos algébricos, vejamos que: $S_{3}(n+2)=S_{3}(n+1)+(n+2)$, subtraindo a primeira da segunda fórmula, observamos que: 
$S_{3}(n+2)-S_{3}(n+1)=S_{3}(n+1)-S_{3}(n)+(n+2)-(n+1)$

$S_{3}(n+2)-S_{3}(n+1)=S_{3}(n+1)-S_{3}(n)+1$

$S_{3}(n+2)=2 S_{3}(n+1)-S_{3}(n)+1$

Agora usaremos $S_{3}(n+3)=S_{3}(n+2)+(n+3)$ e, subtraímos $S_{3}(n+2)=2 S_{3}(n+1)-S_{3}(n)+1$ com isso:

$S_{3}(n+3)-S_{3}(n+2)=S_{3}(n+2)-2 S_{3}(n+1)+S_{3}(n)+(n+3)-1$

$S_{3}(n+3)=2 S_{3}(n+2)-2 S_{3}(n+1)+S_{3}(n)+(n+2)$

Com isso, usando o mesmo procedimento, podemos subtrair $S_{3}(n+2)=S_{3}(n+1)+(n+2)$ de $S_{3}(n+3)=2 S_{3}(n+2)-2 S_{3}(n+1)+S_{3}(n)_{n}+(n+2)$ visando eliminar o termo $(n+2)$, portanto: $S_{3}(n+3)-S_{3}(n+2)=2 S_{3}(n+2)-2 S_{3}(n+1)-S_{3}(n+1)+S_{3}(n)+(n+2)-(n+2), \quad$ ou $\quad$ seja $S_{3}(n+3)=3 S_{3}(n+2)-3 S_{3}(n+1)+S_{3}(n)$ que é uma recorrência linear homogênea de $3^{\text {a }}$ ordem, reescrevendo essa recorrência igualando a zero temos $S_{3}(n+3)-3 S_{3}(n+2)+3 S_{3}(n+1)-S_{3}(n)=0$. Veja que comparando com a função genérica, $\frac{a_{0}+a_{1} x+a_{2} x^{2}+\cdots+a_{n-1} x^{n-1}}{b_{0}+b_{1} x+b_{2} x^{2}+\cdots+b_{n} x^{n}}=c_{0}+c_{1} x+c_{2} x^{2}+\cdots+c_{n} x^{n}$ como temos $n=3$ reescrevendo com os valores temos $\frac{a_{0}+a_{1} x+a_{2} x^{2}}{b_{0}+b_{1} x+b_{2} x^{2}+b_{3} x^{3}}=c_{0}+c_{1} x+c_{2} x^{2}+c_{3} x^{3}$, também sabemos que os números $b_{0}, b_{1}, b_{2}, \ldots, b_{n}$ são coeficientes da recorrência então determinaremos $g(x)=b_{0}+b_{1} x+b_{2} x^{2}+b_{3} x^{3}$ com os valores de $S_{3}(n+3)-3 S_{3}(n+2)+3 S_{3}(n+1)-S_{3}(n)=0$, que agora podemos escrever $c_{n+3}-3 c_{n+2}+3 c_{n+1}-c_{n}=0$ assim sendo, temos que: $b_{0}=1, b_{1}=-3, b_{2}=3$ e $b_{3}=-1$, com isso $g(x)=1-3 x+3 x^{2}-x^{3}$ ou seja $g(x)=(1-x)^{3}$.

Para os valores iniciais, como se trata de uma recorrência linear de $3^{\mathrm{a}}$ ordem, deveremos ter os três primeiros valores, ou valores iniciais, recorremos para isso, usando a figura 1, logo, $S_{3}(1)=1, S_{3}(2)=3$ e $S_{3}(3)=6$ que agora podemos escrever $c_{0}=1, c_{1}=3$ e $c_{2}=6$. Por fim, encontraremos os valores dos coeficientes do polinômio $a_{0}, a_{1} \mathrm{e} a_{2}$ conforme as igualdades $a_{0}=b_{0} c_{0}, a_{1}=b_{0} c_{1}+b_{1} c_{0}, a_{2}=b_{0} c_{2}+b_{1} c_{1}+b_{2} c_{0}$ então segue que:

$a_{0}=1 \cdot 1=1$

$a_{1}=1 \cdot 3-3 \cdot 1=0$

$a_{2}=1 \cdot 6-3 \cdot 3+3 \cdot 1=0$

Então temos que $f(x)=1, \log$ a função geradora para os números triangulares é: $\frac{f(x)}{g(x)}=\frac{1}{(1-x)^{3}}=1+3 x+6 x^{2}+\cdots+S_{3}(n) x^{n-1}$

Multiplicando a igualdade por $x ; \frac{x \cdot f(x)}{g(x)}=\frac{x}{(1-x)^{3}}=1 x+3 x^{2}+6 x^{3}+\cdots+S_{3}(n) x^{n}$.

Nos passos posteriores, buscaremos a generalização dos números poligonais definidos por Deza, E. e Deza, M. M. (2012) como $S_{m}(n)$

Agora apresentaremos o mesmo procedimento para encontrar a função geradora dos números quadrados, $(1,4,9,16, \ldots)$ 


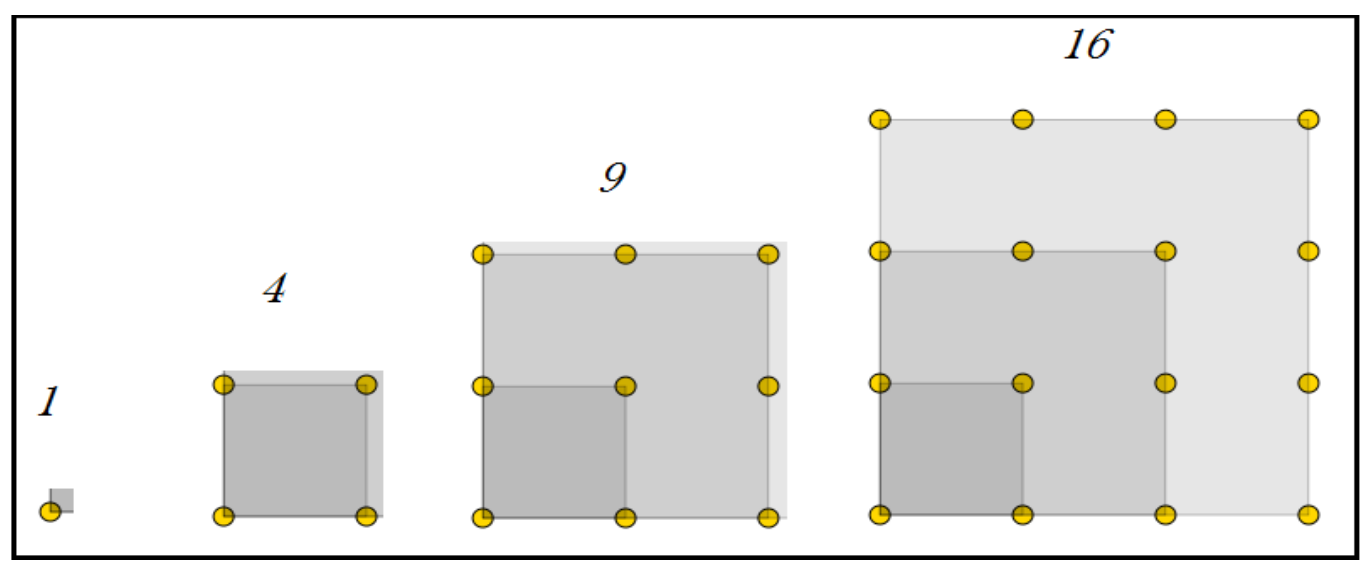

Figura 2 - Os quatro primeiros número quadrados

Fonte: elaboração dos autores

Onde $S_{4}(1)=1, S_{4}(2)=4, S_{4}(3)=9$ e $S_{4}(n)=n^{2}$ com $n \in \mathbb{N}$, para isso necessitaremos da relação de recorrência homogênea, usando os mesmos procedimentos anteriores temos:

$S_{4}(1)=1$,

$S_{4}(2)=4=1+3=S_{4}(1)+2 \cdot 1+1$

$S_{4}(3)=9=4+5=S_{4}(2)+2 \cdot 2+1$

$\vdots$

$S_{4}(n+1)=S_{4}(n)+2(n)+1$

Logo:

$S_{4}(n+1)=S_{4}(n)+2 n+1, S_{4}(n+2)=S_{4}(n+1)+2(n+1)+1$

$S_{4}(n+2)-S_{4}(n+1)=S_{4}(n+1)-S_{4}(n)+2$

$S_{4}(n+2)=2 S_{4}(n+1)-S_{4}(n)+2$.

$S_{4}(n+3)=S_{4}(n+2)+2(n+2)+1$

$S_{4}(n+3)-S_{4}(n+2)=S_{4}(n+2)-2 S_{4}(n+1)+S_{4}(n)+2 n+3$

$S_{4}(n+3)=2 S_{4}(n+2)-2 S_{4}(n+1)+S_{4}(n)+2 n+3$.

$S_{4}(n+3)-S_{4}(n+2)=2 S_{4}(n+2)-3 S_{4}(n+1)+S_{4}(n)$

$S_{4}(n+3)=3 S_{4}(n+2)-3 S_{4}(n+1)+S_{4}(n)$.

$S_{4}(n+3)-3 S_{4}(n+2)+3 S_{4}(n+1)-S_{4}(n)=0$

Novamente verificamos uma recorrência de $3^{\mathrm{a}}$ ordem, logo podemos usar a mesma fórmula canônica $\frac{a_{0}+a_{1} x+a_{2} x^{2}}{b_{0}+b_{1} x+b_{2} x^{2}+b_{3} x^{3}}=c_{0}+c_{1} x+c_{2} x^{2}+c_{3} x^{3}$, para encontrar os termos do polinômio $S_{4}(n+3)-3 S_{4}(n+2)+3 S_{4}(n+1)-S_{4}(n)$, sendo $S_{4}(n)=c_{0}, S_{4}(n+1)=c_{1}, \ldots$ percebemos que os valore de $b_{i}, \mathrm{i} \in \square$ não se alteram em relação aos números triangulares e a única diferença são os valores iniciais $c_{0}=1, c_{1}=4$ e $c_{2}=9$, o que podemos concluir que:

$a_{0}=1 \cdot 1=1$

$a_{1}=1 \cdot 4-3 \cdot 1=1$

$a_{2}=1 \cdot 9-3 \cdot 4+3 \cdot 1=0$ 
Assim, podemos definir a função geradora $\frac{1+x}{(1-x)^{3}}=1+4 x+9 x^{2}+\cdots+\left(S_{4}(n)\right) x^{n-1}$

Ou ainda: $\frac{x(1+x)}{(1-x)^{3}}=1 x+4 x^{2}+9 x^{3}+\cdots+\left(S_{4}(n)\right) x^{n},|x|<1$.

Para obtermos a função geradora dos números pentagonais, buscaremos, além de definir as funções $f(x)$ e $g(x)$, encontrar uma regularidade que possa nos fornecer meios de generalização da família dos números $m$-gonais, para tanto observamos a figura que nos apresenta os primeiros números pentagonais:

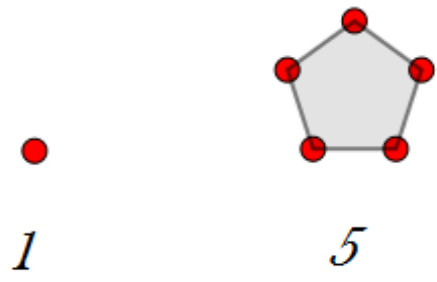

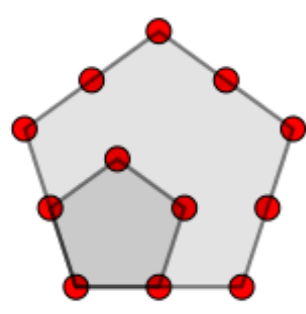

12

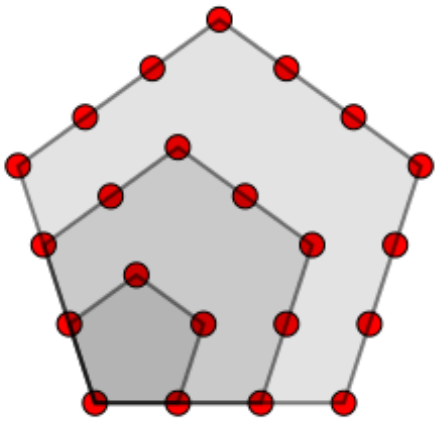

22

Figura 3 - Os quatro primeiros números pentagonais

Fonte: elaboração dos autores

Temos os primeiros números pentagonais apresentados por $S_{5}(1)=1, S_{5}(2)=5, S_{5}(3)=12$ e $S_{5}(4)=22$, e observamos que:

$S_{5}(1)=1$

$S_{5}(2)=5=1+4=S_{5}(1)+3 \cdot 1+1$

$S_{5}(3)=12=5+7=S_{5}(2)+3 \cdot 2+1$

$S_{5}(4)=22=12+10=S_{5}(3)+3 \cdot 3+1$

:

$S_{5}(n+1)=S_{5}(n)+3 \cdot n+1[1]$

Observamos, assim como nos números quadrados e triangulares, que necessitaremos de alguns processor algébricos para determinar uma recorrência linear homogênea, tal processo é semelhante aos já usados anteriormente nas sequências citadas acima. Assim, observamos que os números pentagonais podem ser descritos como $S_{5}(n+2)=S_{5}(n+1)+3 \cdot(n+1)+1$ [2], também podemos apresentar tais números como $S_{5}(n+3)=S_{5}(n+2)+3 \cdot(n+2)+1$ [3] , fazendo [2]-[1] temos:

$S_{5}(n+2)-S_{5}(n+1)=S_{5}(n+1)-S_{5}(n)+3 n+3+1-3 n-1$

$S_{5}(n+2)=2 S_{5}(n+1)-S_{5}(n)+3[4]$

Com [3]-[2] temos:

$S_{5}(n+3)-S_{5}(n+2)=S_{5}(n+2)-S_{5}(n+1)+3 \cdot(n+2)+1-3 \cdot(n+1)-1$

$S_{5}(n+3)=2 S_{5}(n+2)-S_{5}(n+1)+3 \quad[5]$

Por fim, temos que [5]-[4] é igual a: 
$S_{5}(n+3)-S_{5}(n+2)=2 S_{5}(n+2)-2 S_{5}(n+1)-S_{5}(n+1)+S_{5}(n)+3-3$

$S_{5}(n+3)=3 S_{5}(n+2)-3 S_{5}(n+1)+S_{5}(n)$

$S_{5}(n+3)-3 S_{5}(n+2)+3 S_{5}(n+1)-S_{5}(n)=0$

Percebemos que se trata de uma recorrência com os mesmos coeficientes das recorrências encontradas anteriormente, por isso podemos definir a função $g(x)=(1-x)^{3}$, com os valores de $b_{0}=1, b_{1}=-3, b_{2}=3 \mathrm{e} \quad b_{3}=-1$, também temos que $S_{5}(1)=c_{0}=1, \quad S_{5}(2)=c_{1}=5$, $S_{5}(3)=c_{2}=12$ e $S_{5}(4)=c_{3}=22$, desse modo, os valores dos coeficientes de $f(x)$ podem ser determinados como: $a_{0}=b_{0} c_{0}=1 \cdot 1=1, a_{1}=b_{0} c_{1}+b_{1} c_{0}=1 \cdot 5-3 \cdot 1=2$ e $a_{2}=b_{0} c_{2}+b_{1} c_{1}+b_{2} c_{1}$ ou seja, $a_{2}=1 \cdot 12-3 \cdot 5+3 \cdot 1=0 \operatorname{logo} f(x)=1+2 x$ e temos a função geradora dos números pentagonais defina como $\frac{1+2 x}{(1-x)^{3}}=1+5 x+12 x^{2}+22 x^{3}+\cdots+S_{5}(n) x^{n-1}$ ou ainda: $\frac{x(1+2 x)}{(1-x)^{3}}=1 x+5 x^{2}+12 x^{3}+22 x^{4}+\cdots+S_{5}(n) x^{n}$

De um modo geral temos:

$S_{3}(n+1)=S_{3}(n)+n+1$

$S_{4}(n+1)=S_{4}(n)+2 n+1$

$S_{5}(n+1)=S_{5}(n)+3 n+1$

:

$S_{m}(n+1)=S_{m}(n)+(m-2)+1$

Podemos analisar melhor essa afirmação por meio da figura:
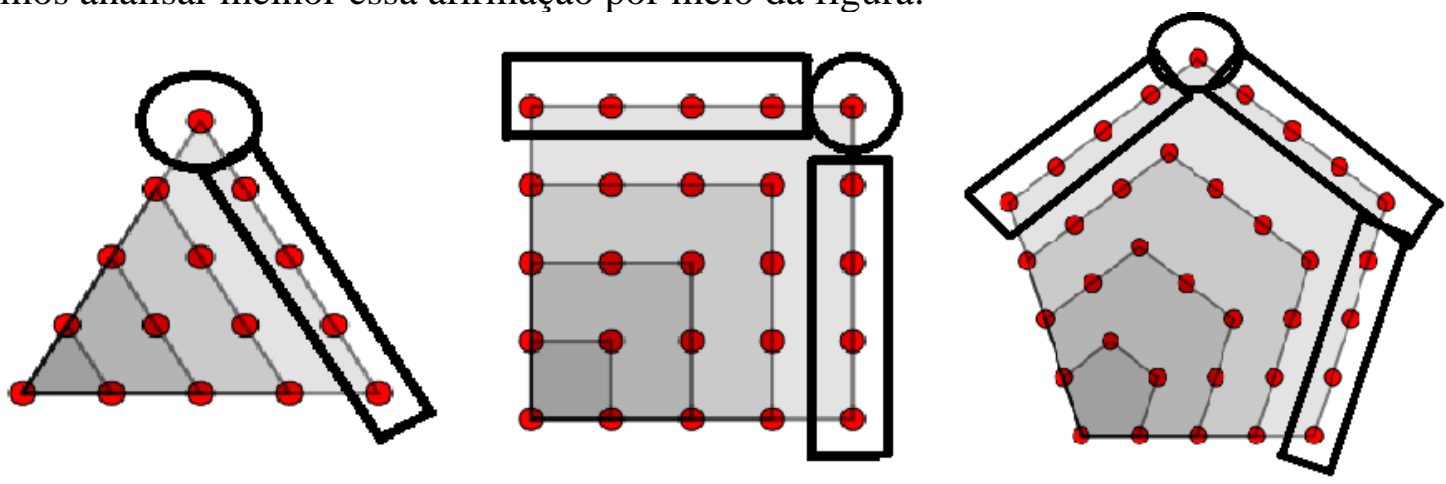

Figura 4 - Relação de um número poligonal com o seu anterior

Fonte: elaboração dos autores

Perceba que para formar um número poligonal a partir do segundo, precisamos do seu anterior e adicionamos a este, blocos que representam o número de pontos do vértice do polígono anterior. $\mathrm{O}$ número de blocos é sempre o número de vértices do polígono menos dois, isso porque usamos sempre dois vértices da figura anterior, ou seja $m-2$ vértices, depois disto, para completar o próximo número poligonal, ainda precisamos de um ponto, como mostra a figura acima.

Agora precisamos determinar os primeiros números poligonais, obsevando as regularidades vistas nos números triangulares, quadrados e pentagonais, por definição dos números figurados temos que $S_{m}(1)=1$, para determinarmos $S_{m}(2)$ perceba que este tem o número de pontos coincidentes ao número de vértices da figura que está se formando, deste modo $S_{m}(2)=m$ e para 
determinarmos $S_{m}(3)$, usaremos o fato apresentado pela figura 4 , assim temos que $S_{m}(3)=S_{m}(2)+(m-2) \cdot 2+1=m+(m-2) \cdot 2+1=3 m-3$.

Assim, a função geradora para o $n$-ésimo número $m$-gonal pode ser encontrada usando:

$S_{m}(n+1)=S_{m}(n)+(m-2) n+1[1], S_{m}(n+2)=S_{m}(n+1)+(m-2)(n+1)+1$ [2] e $S_{m}(n+3)=S_{m}(n+2)+(m-2)(n+2)+1$ [3], fazendo [2] $-[1]$ temos:

$S_{m}(n+2)-S_{m}(n+1)=S_{4}(n+1)-S_{4}(n)+m-2$

$S_{m}(n+2)=2 S_{m}(n+1)-S_{m}(n)+m-2 \quad[4]$

Usando [3]-[2] temos:

$S_{m}(n+3)-S_{m}(n+2)=S_{4}(n+2)-S_{4}(n+1)+m-2$

$S_{m}(n+3)=2 S_{m}(n+2)-S_{m}(n+1)+m-2[5]$

Por fim, calculando [5]-[4]:

$S_{m}(n+3)-S_{m}(n+2)=2 S_{m}(n+2)-2 S_{m}(n+1)-S_{m}(n+1)+S_{m}(n)+(m-2)-(m-2)$

$S_{m}(n+3)=3 S_{m}(n+2)-3 S_{m}(n+1)+S_{m}(n)$

Ou ainda:

$$
S_{m}(n+3)-3 S_{m}(n+2)+3 S_{m}(n+1)-S_{m}(n)=0
$$

Como os valores dos coeficientes da função $g(x)$, são os mesmo para todos os números poligonais, pois usamos sempre o mesmo processo para encontrar a recorrência linear homogênea para os números poligonais, temos que $g(x)=(1-x)^{3}$, ainda temos os três primeiros números poligonais, assim $S_{m}(1)=c_{0}=1, S_{m}(2)=c_{1}=m$ e $S_{m}(3)=c_{2}=3 m-3$, assim os valores dos coeficientes de $f(x)$ são:

$$
\begin{aligned}
& a_{0}=b_{0} c_{0}=1 \cdot 1=1 \\
& a_{1}=b_{0} c_{1}+b_{1} c_{0}=1 \cdot m-3 \cdot 1=m-3 \\
& a_{2}=b_{0} c_{2}+b_{1} c_{1}+b_{2} c_{0}=1 \cdot(3 m-3)-3 m+3=0
\end{aligned}
$$

Isso significa que $f(x)=1+(m-2) x$, assim, a função geradora para qualquer número poligonal é dada por $\frac{1+(m-3) x}{(1-x)^{3}}=S_{m}(1)+S_{m}(2) x+S_{m}(3) x^{2}+\ldots+S_{m}(n) x^{n-1}$ ou ainda:

$$
\frac{x(1+(m-3) x)}{(1-x)^{3}}=S_{m}(1) x+S_{m}(2) x^{2}+S_{m}(3) x^{3}+\ldots+S_{m}(n) x^{n}
$$

Assim definimos um função geradora para a família de sequências dos números poligonais, em particular, definimos a função geradora para $m=3$, dos números triangulares, para $m=4$, dos número quadrados, para $m=5$, dos números pentagonais, $m=6$ para os números hexagonais e assim por diante.

\section{As funções geradoras dos análogos uni, tri e tetradimensionais dos números triangulares}

Na geometria, definimos por simplexo, todos os análogos $k$-dimensionais dos triângulos, ou seja, toda figura que possui $k+1$ vértices dada uma dimensão $k$. Assim trataremos nesse capítulo das funções geradoras dos números lineares, triangulares, tetraédricos (análogo tridimensional do triangulo), números de pentatope (análogos da quarta dimensão dos triângulos), e assim sucessivamente. 
Definimos por números figurados lineares $S_{3}^{1}$, a sequência de pontos que formam os gnômons dos números triangulares, ou seja, o termos nos quais devemos somar um número triangular para que se obtenha o próximo.

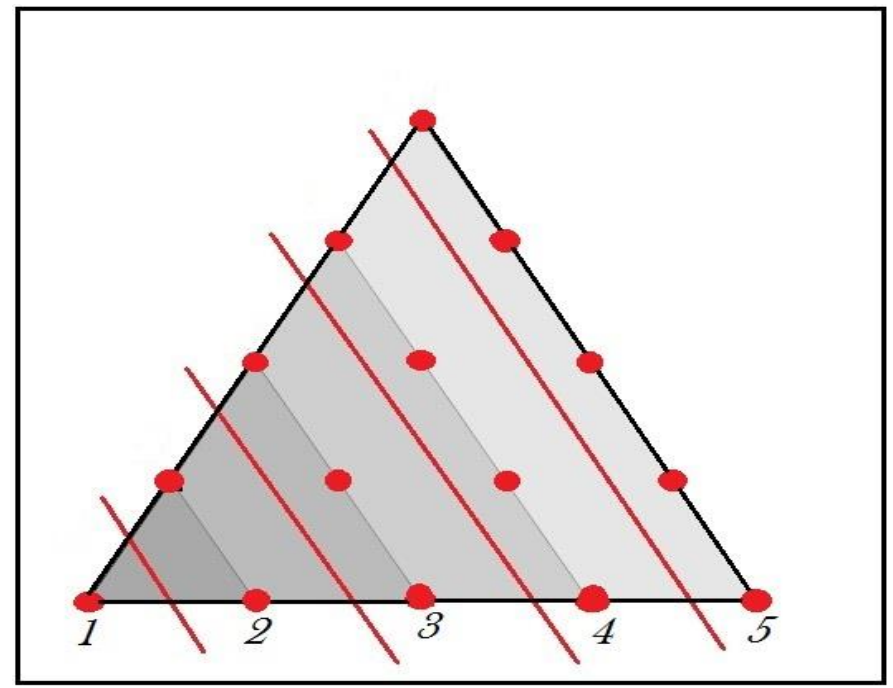

Figura 5 - Números triangulares a partir do gnômom

Fonte: elaboração dos autores

Esses números se relacionam com os triângulos justamente porque os números triangulares são formados pela soma sucessiva de números lineares ou números naturais, assim os primeiros números lineares são: $S_{3}^{1}(1)=1, S_{3}^{1}(2)=2, \ldots, S_{3}^{1}(n)=n$, para $n \in \square$. Esta fórmula não nos permite encontrar a função geradora a princípio, pois seu $n$-ésimo termo ainda não está em função dos anteriores, para isso temos que:

$S_{3}^{1}(1)=1$

$S_{3}^{1}(2)=2=1+1=S_{3}^{1}(1)+1$

$S_{3}^{1}(3)=3=2+1=S_{3}^{1}(2)+1$

:

$S_{3}^{1}(n)=n=n-1+1=S_{3}^{1}(n-1)+1$

$S_{3}^{1}(n+1)=n+1=n+1=S_{3}^{1}(n)+1$

Essa recorrência ainda não nos fornece os termos em relação aos anteriores, porém se observarmos que: $S_{3}^{1}(n+2)-S_{3}^{1}(n+1)=S_{3}^{1}(n+1)+1-S_{3}^{1}(n)-1$ assim, $S_{3}^{1}(n+2)=2 S_{3}^{1}(n+1)-S_{3}^{1}(n)$.

Para a função geradora basta observar que essa é uma recorrência de segunda ordem com os termos iniciais $S_{3}^{1}(1)=1$ e $S_{3}^{1}(2)=2$, então temos a forma genérica para a segunda ordem descrita como $\frac{a_{0}+a_{1} x}{b_{0}+b_{1} x+b_{2} x^{2}}=c_{0}+c_{1} x+c_{2} x^{2}$ e $a_{0}=b_{0} c_{0}, a_{1}=b_{0} c_{1}+b_{1} c_{0}, a_{2}=b_{0} c_{2}+b_{1} c_{1}+b_{2} c_{0}$ temos ainda que a recorrência pode ser escrita como $S_{3}^{1}(n+2)-2 S_{3}^{1}(n+1)+S_{3}^{1}(n)=0$ ou ainda $c_{n+2}-2 c_{n+1}+c_{n}=0$ o que nos fornece os valores de $b_{0}=1, b_{1}=-2$ e $b_{2}=1$, definindo assim o polinômio $g(x)=1-2 x+x^{2}=(1-x)^{2}$. Para $f(x)=a_{0}+a_{1} x$, com os valores iniciais da sequência sendo $c_{0}=1$ e $c_{1}=2$, daí encontramos $a_{0}=1$ e $a_{1}=1 \cdot 2-2 \cdot 1=0$, o que nos fornece a 
função geradora $\frac{f(x)}{g(x)}=\frac{1}{(1-x)^{2}}=1+2 x+3 x^{2}+\cdots+n x^{n-1} \quad$ com $\quad x<1 \quad$ ou $\quad$ ainda $\frac{x \cdot f(x)}{g(x)}=\frac{x}{(1-x)^{2}}=x+2 x^{2}+3 x^{3}+\cdots+n x^{n}$ com $x<1$.

A próxima sequência será a dos números tetraédricos, pois a função geradora da sequência de números triangulares já foi descrita na seção anterior e dada por $\frac{x}{(1-x)^{3}}$. Os números tetraédricos são dados pelas somas sucessivas dos $n$ números triangulares, assim:

$S_{3}^{3}(1)=S_{3}^{2}(1)=1$

$S_{3}^{3}(2)=S_{3}^{2}(1)+S_{3}^{2}(2)=1+3=4$

$S_{3}^{3}(1)=S_{3}^{2}(1)+S_{3}^{2}(2)+S_{3}^{2}(3)=1+3+6=10$

$S_{3}^{3}(n)=\sum_{i=1}^{n} S_{3}^{3}(i)$

Os números tetraédricos ainda podem ser definidos em relação ao seu anterior segundo a recorrência $S_{3}^{3}(n+1)=S_{3}^{3}(n)+S_{3}^{2}(n+1)$, que relacionam também os números triangulares, assim, para encontrarmos uma recorrência que não relacione os números triangulares, teremos que usar a fórmula do termos geral de tais números, em outras palavras reescrevemos a fórmula como $S_{3}^{3}(n+1)=S_{3}^{3}(n)+\frac{(n+1)(n+2)}{2}$, buscaremos com isso, encontrar uma recorrência linear homogênea, ou seja que possua apenas termos em função dos números tetraédricos anteriores. Incorremos para esse objetivo com algumas fórmulas para facilitar a percepção:

$S_{3}^{3}(n+1)=S_{3}^{3}(n)+\frac{(n+1)(n+2)}{2}[1]$

$S_{3}^{3}(n+2)=S_{3}^{3}(n+1)+\frac{(n+2)(n+3)}{2}$

$S_{3}^{3}(n+3)=S_{3}^{3}(n+2)+\frac{(n+3)(n+4)}{2}$

$S_{3}^{3}(n+4)=S_{3}^{3}(n+3)+\frac{(n+4)(n+5)}{2}$

Como isso e através de procedimento algébricos simples, buscaremos encontrar tal recorrência calculando [2]-[1], temos:

$S_{3}^{3}(n+2)-S_{3}^{3}(n+1)=S_{3}^{3}(n+1)+\frac{(n+2)(n+3)}{2}-\left(S_{3}^{3}(n)+\frac{(n+1)(n+2)}{2}\right)$

$S_{3}^{3}(n+2)=S_{3}^{3}(n+1)+S_{3}^{3}(n+1)-S_{3}^{3}(n)+\frac{(n+2)}{2} \cdot(n+3-n-1)$

$S_{3}^{3}(n+2)=2 S_{3}^{3}(n+1)-S_{3}^{3}(n)+(n+2)$

Do mesmo modo, para [3]-[2] temos $S_{3}^{3}(n+3)=2 S_{3}^{3}(n+2)-S_{3}^{3}(n+1)+(n+3)$ [6] para [4]-[3] teremos $S_{3}^{3}(n+4)=2 S_{3}^{3}(n+3)-S_{3}^{3}(n+2)+(n+4)$ [7] , para [6]-[5]: 
$S_{3}^{3}(n+3)-S_{3}^{3}(n+2)=2 S_{3}^{3}(n+2)-S_{3}^{3}(n+1)+(n+3)-\left(2 S_{3}^{3}(n+1)-S_{3}^{3}(n)+(n+2)\right)$

$S_{3}^{3}(n+3)=3 S_{3}^{3}(n+2)-3 S_{3}^{3}(n+1)+S_{3}^{3}(n)+1[8]$

para [7]-[6]:

$S_{3}^{3}(n+4)-S_{3}^{3}(n+3)=2 S_{3}^{3}(n+3)-S_{3}^{3}(n+2)+(n+4)-\left(2 S_{3}^{3}(n+2)-S_{3}^{3}(n+1)+(n+3)\right)$

$S_{3}^{3}(n+4)=3 S_{3}^{3}(n+3)-3 S_{3}^{3}(n+2)+S_{3}^{3}(n+1)+1[9]$

Por fim faremos [9]-[8]:

$S_{3}^{3}(n+4)-S_{3}^{3}(n+3)=3 S_{3}^{3}(n+3)-3 S_{3}^{3}(n+2)+S_{3}^{3}(n+1)+1-\left(3 S_{3}^{3}(n+2)-3 S_{3}^{3}(n+1)\right.$

$\left.+S_{3}^{3}(n)+1\right)$

$S_{3}^{3}(n+4)=4 S_{3}^{3}(n+3)-6 S_{3}^{3}(n+2)+4 S_{3}^{3}(n+1)-S_{3}^{3}(n)$

E assim, a recorrência linear homogênea dos números tetraédricos é dada por:

$$
S_{3}^{3}(n+4)-4 S_{3}^{3}(n+3)+6 S_{3}^{3}(n+2)-4 S_{3}^{3}(n+1)+S_{3}^{3}(n)=0
$$

Como percebemos, essa recorrência é de quarta ordem ou seja, necessitaremos dos três primeiros valores da sequência, para isso a observamos o comportamento desta seguindo a figura:

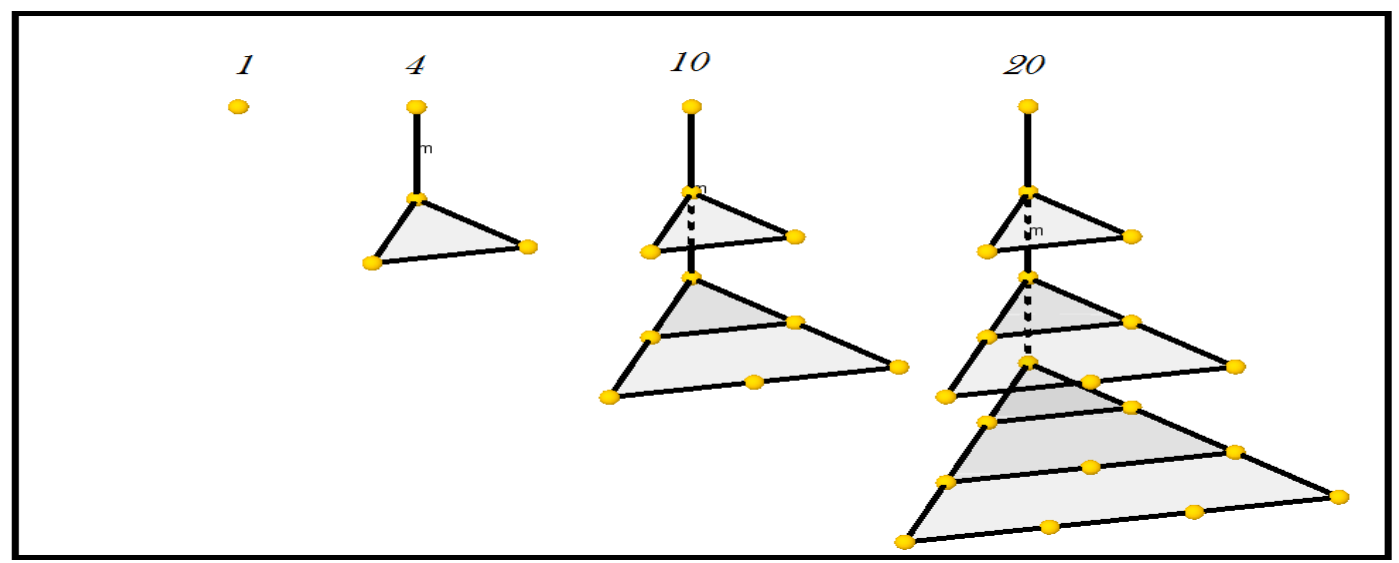

Figura 6 - Os quatro primeiros números tetraédricos

Fonte: elaboração dos autores

A figura apresenta os quatro primeiros números piramidais triangulares ou números tetraédricos, esses números são formados a partir do empilhamento dos $n$ primeiros números triangulares ou seja, o terceiro número tetraédrico é a soma dos três primeiros números triangulares, vale lembrar que assim como o triângulo é a figura mais simples que podemos formar unindo o mínimo possível de segmentos de reta em duas dimensões, o tetraedro, por sua vez é o sólido geométrico mais simples em três dimensões.

Dada esta breve explicação acerca dos números tetraédricos, aplicaremos a função genérica $\frac{f(x)}{g(x)}=\frac{a_{0}+a_{1} x+a_{2} x^{2}+a_{3} x^{3}}{b_{0}+b_{1} x+b_{2} x^{2}+b_{3} x^{3}+b_{4} x^{4}}=c_{0}+c_{1} x+c_{2} x^{2}+\ldots$ como visto nas outras seções, para determinar a função geradora da sequência desejada. Observamos que podemos determinar os valores dos coeficientes de $g(x)$, pois são os mesmos coeficientes da recorrência, temos então $b_{0}=1, b_{1}=-4, b_{2}=6, b_{3}=-4$ e $b_{4}=1$, daí temos $g(x)=1-4 x+6 x^{2}-4 x^{3}-x^{4}=(1-x)^{4}$, para os valores dos coeficientes de $f(x)$, encontramos: 
$a_{0}=b_{0} c_{0}=1 \cdot 1=1$

$a_{1}=b_{0} c_{1}+b_{1} c_{0}=1 \cdot(-4)+4 \cdot 1=0$

$a_{2}=b_{0} c_{2}+b_{1} c_{1}+b_{2} c_{0}=1 \cdot 10-4 \cdot 4+6 \cdot 1=0$

$a_{3}=b_{0} c_{3}+b_{1} c_{2}+b_{2} c_{1}+b_{3} c_{0}=1 \cdot 20-4 \cdot 10+6 \cdot 4-4 \cdot 1=0$

que nos fornece $f(x)=1$, sendo a função geradora dos números tetraédricos dada por:

$\frac{f(x)}{g(x)}=\frac{1}{(1-x)^{4}}=1+4 x+10 x^{2}+\ldots+\left(S_{3}^{3}(n)\right) x^{n-1}$

$\frac{x \cdot f(x)}{g(x)}=\frac{x}{(1-x)^{4}}=x+4 x^{2}+10 x^{3}+\ldots+\left(S_{3}^{3}(n)\right) x^{n}$

Para os números de pentatope, observamos que Deza e Deza, 2012, realiza um procedimento que podemos usar para generalizar as funções geradoras dos números hipertetraédricos, basta lembrar que dada uma dimensão $k+1$, o n-ésimo número é dado pela soma dos $n$ primeiros números da dimensão $k$, juntado isto ao fato da recursividade dos números da família dos simplexos, os números de pentatope são definidos pela sequência $(1,5,15,35,70, \ldots)$. Usando os recursos da recursividade dimensional, observamos que:

$S_{3}^{4}(1)=1$

$S_{3}^{4}(2)=5=1+4=S_{3}^{4}(1)+S_{3}^{3}(2)$

$S_{3}^{4}(3)=15=5+10=S_{3}^{4}(2)+S_{3}^{3}(3)$

$S_{3}^{4}(4)=35=15+20=S_{3}^{4}(3)+S_{3}^{3}(4)$

:

$S_{3}^{4}(n+1)=S_{3}^{4}(n)+S_{3}^{3}(n+1)$

Para definirmos a função geradora dos números de pentatope, tomaremos subtrações sucessivas bem como as realizadas nos números das seções anteriores, assim $S_{3}^{4}(n+2)-S_{3}^{4}(n+1)=S_{3}^{4}(n+1)-S_{3}^{4}(n)+\left(S_{3}^{3}(n+2)-S_{3}^{3}(n+1)\right)$, observe que o temos em parênteses, ou seja $S_{3}^{3}(n+2)-S_{3}^{3}(n+1)$ é a subtração de dois números tetraédricos seguidos, que pela relação de recursividade $S_{3}^{3}(n+2)=S_{3}^{3}(n+1)+S_{3}(n+2)$, logo $S_{3}^{3}(n+2)-S_{3}^{3}(n+1)=S_{3}(n+2)$ então temos que:

$S_{3}^{4}(n+2)-S_{3}^{4}(n+1)=S_{3}^{4}(n+1)-S_{3}^{4}(n)+S_{3}(n+2)$

$S_{3}^{4}(n+2)=2 S_{3}^{4}(n+1)-S_{3}^{4}(n)+S_{3}(n+2) \quad[1]$

Tomamos os mesmo procedimento até que os termos das dimensões inferiores a quarta, não estejam mais presentes e assim obteremos a recorrência linear e homogênea dessa sequência: $S_{3}^{4}(n+3)-S_{3}^{4}(n+2)=S_{3}^{4}(n+2)-S_{3}^{4}(n+1)+\left(S_{3}^{3}(n+3)-S_{3}^{3}(n+2)\right)$

$S_{3}^{4}(n+3)=2 S_{3}^{4}(n+2)-S_{3}^{4}(n+1)+S_{3}(n+3)$

Operando [2]-[1] obtemos:

$S_{3}^{4}(n+3)-S_{3}^{4}(n+2)=2 S_{3}^{4}(n+2)-S_{3}^{4}(n+1)+S_{3}(n+3)-\left(2 S_{3}^{4}(n+1)-S_{3}^{4}(n)+S_{3}(n+2)\right)$

$S_{3}^{4}(n+3)=3 S_{3}^{4}(n+2)-S_{3}^{4}(n+1)+S_{3}(n+3)-\left(2 S_{3}^{4}(n+1)-S_{3}^{4}(n)+S_{3}(n+2)\right)$ 


$$
\begin{aligned}
& S_{3}^{4}(n+3)=3 S_{3}^{4}(n+2)-3 S_{3}^{4}(n+1)+S_{3}^{4}(n)+\left(S_{3}(n+3)-S_{3}(n+2)\right) \\
& S_{3}^{4}(n+3)=3 S_{3}^{4}(n+2)-3 S_{3}^{4}(n+1)+S_{3}^{4}(n)+(n+3) \\
& S_{3}^{4}(n+4)-S_{3}^{4}(n+3)=S_{3}^{4}(n+3)-S_{3}^{4}(n+2)+\left(S_{3}^{3}(n+4)-S_{3}^{3}(n+3)\right) \\
& S_{3}^{4}(n+4)=2 S_{3}^{4}(n+3)-S_{3}^{4}(n+2)+\left(S_{3}(n+4)\right)
\end{aligned}
$$

Para [4]-[2], temos:

$S_{3}^{4}(n+4)-S_{3}^{4}(n+3)=2 S_{3}^{4}(n+3)-S_{3}^{4}(n+2)+\left(S_{3}(n+4)\right)-\left(2 S_{3}^{4}(n+2)-S_{3}^{4}(n+1)\right)$

$-\left(S_{3}(n+3)\right)$

$S_{3}^{4}(n+4)=3 S_{3}^{4}(n+3)-3 S_{3}^{4}(n+2)+S_{3}^{4}(n+1)+\left(S_{3}(n+4)-S_{3}(n+3)\right)$

$S_{3}^{4}(n+4)=3 S_{3}^{4}(n+3)-3 S_{3}^{4}(n+2)+S_{3}^{4}(n+1)+(n+4)$

Para [5]-[3]:

$S_{3}^{4}(n+4)-S_{3}^{4}(n+3)=3 S_{3}^{4}(n+3)-3 S_{3}^{4}(n+2)+S_{3}^{4}(n+1)+(n+4)-3 S_{3}^{4}(n+2)$

$+3 S_{3}^{4}(n+1)-S_{3}^{4}(n)+(n+3)$

$S_{3}^{4}(n+4)=4 S_{3}^{4}(n+3)-6 S_{3}^{4}(n+2)+4 S_{3}^{4}(n+1)-S_{3}^{4}(n)+1 \quad[6]$

$S_{3}^{4}(n+5)-S_{3}^{4}(n+4)=S_{3}^{4}(n+4)-S_{3}^{4}(n+3)+\left(S_{3}^{3}(n+5)-S_{3}^{3}(n+4)\right)$

$S_{3}^{4}(n+5)=2 S_{3}^{4}(n+4)-S_{3}^{4}(n+3)+\left(S_{3}(n+5)\right) \quad$ [7]

Para [7]-[4], obtemos:

$S_{3}^{4}(n+5)-S_{3}^{4}(n+4)=2 S_{3}^{4}(n+4)-S_{3}^{4}(n+3)+\left(S_{3}(n+5)\right)-\left(2 S_{3}^{4}(n+3)-S_{3}^{4}(n+2)\right)$

$-\left(S_{3}(n+4)\right)$

$S_{3}^{4}(n+5)=3 S_{3}^{4}(n+4)-3 S_{3}^{4}(n+3)+S_{3}^{4}(n+2)+\left(S_{3}(n+5)-S_{3}(n+4)\right)$

$S_{3}^{4}(n+5)=3 S_{3}^{4}(n+4)-3 S_{3}^{4}(n+3)+S_{3}^{4}(n+2)+(n+5)$

Para [8]-[5] temos:

$S_{3}^{4}(n+5)-S_{3}^{4}(n+4)=3 S_{3}^{4}(n+4)-3 S_{3}^{4}(n+3)+S_{3}^{4}(n+2)+(n+5)-3 S_{3}^{4}(n+3)+3 S_{3}^{4}(n+2)$

$-S_{3}^{4}(n+1)-(n+4)$

$S_{3}^{4}(n+5)=4 S_{3}^{4}(n+4)-6 S_{3}^{4}(n+3)+4 S_{3}^{4}(n+2)-S_{3}^{4}(n+1)+1$

Por fim, operando [9]-[6], teremos:

$S_{3}^{4}(n+5)-S_{3}^{4}(n+4)=4 S_{3}^{4}(n+4)-6 S_{3}^{4}(n+3)+4 S_{3}^{4}(n+2)-S_{3}^{4}(n+1)+1-\left(4 S_{3}^{4}(n+3)\right.$

$\left.-6 S_{3}^{4}(n+2)+4 S_{3}^{4}(n+1)-S_{3}^{4}(n)+1\right)$

$S_{3}^{4}(n+5)=5 S_{3}^{4}(n+4)-10 S_{3}^{4}(n+3)+10 S_{3}^{4}(n+2)-5 S_{3}^{4}(n+1)+S_{3}^{4}(n)$

Ou ainda:

$$
S_{3}^{4}(n+5)-5 S_{3}^{4}(n+4)+10 S_{3}^{4}(n+3)-10 S_{3}^{4}(n+2)+5 S_{3}^{4}(n+1)-S_{3}^{4}(n)=0
$$

Como já se percebe, essa é uma recorrência de $5^{\mathrm{a}}$ ordem, e sua função geradora genérica é dada por $\frac{f(x)}{g(x)}=\frac{a_{0}+a_{1} x+a_{2} x^{2}+a_{3} x^{3}+a_{4} x^{4}}{b_{0}+b_{1} x+b_{2} x^{2}+b_{3} x^{3}+b_{4} x^{4}+b_{5} x^{5}}=c_{0}+c_{1} x+c_{2} x^{2}+c_{3} x^{3}+c_{4} x^{4}+c_{5} x^{5}+\ldots$, onde podemos representar $S_{3}^{4}(n+5)-5 S_{3}^{4}(n+4)+10 S_{3}^{4}(n+3)-10 S_{3}^{4}(n+2)+5 S_{3}^{4}(n+1)-S_{3}^{4}(n)=0$ por $c_{n+5}-5 c_{n+4}+10 c_{n+3}-10 c_{n+2}+5 c_{n+1}-c_{n}=0$, sendo os coeficientes dessa recorrência, os 
mesmo da função $g(x)$, temos que $b_{0}=1, b_{1}=-5, b_{2}=10, b_{3}=-10, b_{4}=5$ e $b_{5}=1$, os primeiros termos da sequência são $c_{0}=1, c_{1}=5, c_{2}=15, c_{3}=35$ e $c_{4}=70$, os termos da função $f(x)$ são; $a_{0}=b_{0} c_{0}=1 \cdot 1=1, a_{1}=b_{0} c_{1}+b_{1} c_{0}=1 \cdot 5-5 \cdot 1=0, a_{2}=b_{0} c_{2}+b_{1} c_{1}+b_{2} c_{0}=1 \cdot 15-5 \cdot 5+10 \cdot 1=0$ $a_{3}=b_{0} c_{3}+b_{1} c_{2}+b_{2} c_{1}+b_{3} c_{0}=1 \cdot 35-5 \cdot 15+10 \cdot 5-10 \cdot 1=0$ e $\quad a_{4}=\sum_{i=0}^{4} b_{i} c_{4-i}=70-175+150-50+5=0$ assim, podemos definir as funções $f(x)=1, g(x)=1-5 x+10 x^{2}-10 x^{3}+5 x^{4}=(1-x)^{5}$, e a razão $\frac{f(x)}{g(x)}=1+5 x+15 x^{2}+\cdots+\left(S_{3}^{4}(n)\right) x^{n-1}$ ou ainda, a função geradora dos números figurados de pentatope como:

$$
\frac{x}{(1-x)^{5}}=x+5 x^{2}+15 x^{3}+35 x^{4}+\cdots+\left(S_{3}^{4}(n)\right) x^{n}
$$

Com a obtenção das funções geradoras dos análogos uni, bi e tri dimensionais da classe dos simplexos, podemos identificar alguns padrões que nos fornecerão argumentos para uma generalização das sequências dessa classe em dimensões arbitrárias, como por exemplo o fato da função $f(x)$ não alterar seu valor, e de $g(x)$ aumentar sempre em uma unidade o expoente do termo $1-x$, conforme aumentamos para a dimensão imediatamente superior. Tais argumentos são favoráveis para que o processo de generalização seja realizado.

\section{A função geradora dos números hipertetraédricos k- dimensionais}

Para buscarmos generalizar as funções geradoras dos números hipertetraédricos ou simplexos, observamos que $\frac{x}{(1-x)^{5}}=\frac{x}{(1-x)^{4}} \cdot \frac{1}{(1-x)}$, sendo $\frac{x}{(1-x)^{4}}$, a função geradora dos números tetraédricos, e $\frac{1}{(1-x)}$, a função geradora da sequência $(1,1,1,1, \ldots .1)$, vale informar que essa sequência é considerada a zero-dimensional da família dos simplexos pelo fato da soma de seus termos gerarem os números lineares (naturais) que por sua vez, se somados consecutivamente geram os triangulares e assim por diante, sendo assim, observaremos a obtenção da função geradora dos números pentatopes, segundo Deza, E. e Deza, M. M. (2012):

$$
\begin{aligned}
& \frac{x}{(1-x)^{5}}=\left[\left(S_{3}^{3}(1)\right) x+\left(S_{3}^{3}(2)\right) x^{2}+\left(S_{3}^{3}(3)\right) x^{3}+\cdots+\left(S_{3}^{3}(n)\right) x^{n}\right] \cdot\left(1+x+x^{2}+x^{3}+\cdots+x^{n-1}\right) \\
& \frac{x}{(1-x)^{5}}=\left(S_{3}^{3}(1)\right) x+\left(S_{3}^{3}(1)+S_{3}^{3}(2)\right) x^{2}+\left(S_{3}^{3}(1)+S_{3}^{3}(2)+S_{3}^{3}(3)\right) x^{3}+\cdots+\left(\sum_{i=1}^{n} S_{3}^{3}(i)\right) x^{n} \\
& \frac{x}{(1-x)^{5}}=\left(S_{3}^{4}(1)\right) x+\left(S_{3}^{4}(2)\right) x^{2}+\left(S_{3}^{4}(3)\right) x^{3}+\cdots+\left(S_{3}^{4}(n)\right) x^{n} \\
& \frac{x}{(1-x)^{5}}=\sum_{i=1}^{n}\left(S_{3}^{4}(i)\right) x^{n}
\end{aligned}
$$

Partindo dessa observação, e, com as fórmulas dos termos gerais de cada números lineares, triangulares, tetraédricos e pentatopes, podemos definir uma fórmula para os números hipertetraédricos $k$-dimensionais para $k \in \square$, observe que os números lineares (sequência $(1,2,3, \ldots, n)$ 
) ou seja, os simplexos da dimensão 1, possuem o termo geral dado por $S_{3}^{1}=n=\frac{n}{1 !}$, os triangulares são obtidos somando-se os termos sucessivos dos números lineares, ou seja $1+2+3+\cdots+n=\frac{1}{2} n(n+1)$, assim o termos geral dos números triangulares é dado por $S_{3}=\frac{n(n+1)}{2}=\frac{n(n+1)}{2 !}$. Para os números tetraédricos, deveremos somar consecutivamente os $n$ primeiros números triangulares, tomando essa afirmação temos o termo geral desta sequência dado por: $S_{3}^{3}=1+3+6+\cdots+\frac{n(n+1)}{2}=\frac{1(1+1)}{2}+\frac{2(2+1)}{2}+\frac{3(3+1)}{2}+\cdots+\frac{n(n+1)}{2}$, observe que $\frac{1 \cdot(1+1)}{2}+\frac{2 \cdot(2+1)}{2}+\cdots+\frac{n \cdot(n+1)}{2}=\frac{1}{2} \cdot\left((1+2+\cdots n)+\left(1^{2}+2^{2}+\cdots+n^{2}\right)\right), \quad$ ou $\quad$ seja $\frac{1}{2} \cdot\left(\frac{n(n+1)}{2}+\left(1^{2}+2^{2}+\cdots+n^{2}\right)\right)$. Para encontrarmos o valor de $1^{2}+2^{2}+\cdots+n^{2}$, vejamos que (BURTON, 1985) $1^{2}+2^{2}+\ldots+n^{2}=\frac{n(n+1)(2 n+1)}{6}$

Demonstração: observando a figura a seguir, buscaremos relações entre o a soma dos $n$ primeiros números quadrados e um retângulo, completando seus lados.
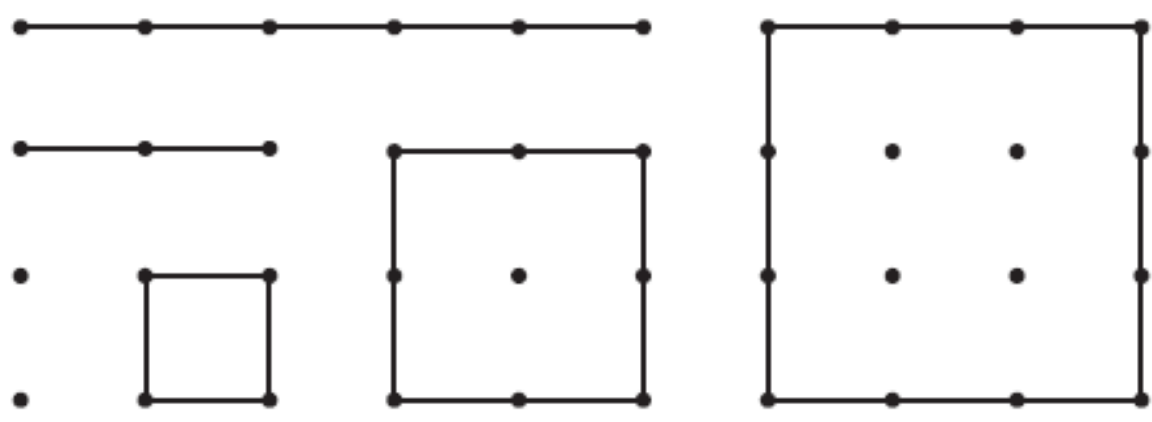

Figura 7 - Esquema de Burton para a soma dos números quadrados.

Fonte: Burton (1985)

Fazendo o teste para os primeiros números quadrados temos que:

Para $n=1$, temos $1^{2}=\frac{1 \cdot 2 \cdot 3}{6}$, para $n=2,1^{2}+2^{2}=\frac{2 \cdot 3 \cdot 5}{6}=5$, e, por fim temos que para $n=3,1^{2}+2^{2}+3^{2}=\frac{3 \cdot 4 \cdot 7}{6}=14$. Recorrendo a figura, observe que para tornar a soma de quadrados iguais ao retângulos formado precisamos adicionar pontos, assim podemos definir que:

$$
\left(1^{2}+2^{2}+3^{2}+\ldots+n^{2}\right)+\left(1+3+6+\ldots+\frac{n(n+1)}{2}\right)=(1+2+3+\ldots+n) \cdot(n+1)
$$

Perceba que o primeiro termo refere-se a soma de quadrados descrita na figura de Burton, os pontos adicionais para se completar o retângulo, são $n$ números triangulares, a base do retângulo formado é a base da soma dos $n$ números naturais e, por fim, a altura do retângulo é 
formado pela altura do $n$-ésimo número quadrado mais uma linha com o $n$-ésimo número triangular, portanto $n+1$ pontos de altura, chamaremos o termo $1^{2}+2^{2}+3^{2}+\ldots+n^{2}$ de $S$, desenvolvendo apenas a soma de números triangulares temos:

$$
\begin{aligned}
& 1+3+6+\ldots+\frac{n(n+1)}{2}=\frac{1 \cdot(1+1)}{2}+\frac{2 \cdot(2+1)}{2}+\frac{3 \cdot(3+1)}{2}+\ldots+\frac{n(n+1)}{2} \\
& \frac{1}{2} \cdot\left(1^{2}+1+2^{2}+2+3^{2}+3+\ldots+n^{2}+n\right)=\frac{1}{2} \cdot\left(1^{2}+2^{2}+3^{2}+\ldots+n^{2}+1+2+3+\ldots+n\right) \\
& \frac{1}{2} \cdot\left(S+\frac{n(n+1)}{2}\right)=\frac{S}{2}+\frac{n(n+1)}{4}
\end{aligned}
$$

Então temos a igualdade a seguir:

$$
S+\frac{S}{2}+\frac{n(n+1)}{4}=\frac{n(n+1)}{2} \cdot(n+1)
$$

Com isso, segue que:

$$
\begin{aligned}
& \frac{3 S}{2}=\frac{n(n+1)^{2}}{2}-\frac{n(n+1)}{4}=\frac{2 n(n+1)^{2}}{4}-\frac{n(n+1)}{4}=\frac{n \cdot(n+1)}{4} \cdot(2 \cdot(n+1)-1)=\frac{n \cdot(n+1)}{4} \cdot(2 n+1) \\
& 3 S=\frac{n \cdot(n+1) \cdot(2 n+1)}{2} \Rightarrow S=\frac{n \cdot(n+1) \cdot(2 n+1)}{6}
\end{aligned}
$$

Superado o problema das somas de quadrados, temos:

$$
\begin{aligned}
& S_{3}^{3}(n)=\frac{1}{2} \cdot\left(\frac{n(n+1)}{2}+\frac{n \cdot(n+1) \cdot(2 n+1)}{6}\right)=\frac{1}{2} \cdot\left(\frac{3 n(n+1)}{6}+\frac{n \cdot(n+1) \cdot(2 n+1)}{6}\right) \\
& =\frac{1}{2} \cdot\left(\frac{n(n+1)(2 n+4)}{6}\right)=\frac{1}{2} \cdot\left(\frac{n(n+1)(n+2)}{3}\right)=\frac{n(n+1)(n+2)}{6}=\frac{n(n+1)(n+2)}{3 !}
\end{aligned}
$$

Assim, podemos propor uma hipótese de que para um certo $k \in \square$, temos $S_{3}^{k}(n)=\frac{n(n+1) \cdots(n+k-1)}{k !}$ e, para provarmos tal fato para $n+1$, temos que:

$S_{3}^{k}(n+1)=S_{3}^{k}(n)+S_{3}^{k-1}(n+1)=\frac{n(n+1) \cdots(n+k-1)}{k !}+\frac{(n+1)(n+2) \cdots(n+1+k-2)}{(k-1) !}$

$S_{3}^{k}(n+1)=S_{3}^{k}(n)+S_{3}^{k-1}(n+1)=\frac{n(n+1) \cdots(n+k-1)}{k !}+\frac{(n+1)(n+2) \cdots(n+k-1) k !}{(k-1) ! k !}$

$S_{3}^{k}(n+1)=\frac{n(n+1) \cdots(n+k-1)}{k !}+\frac{(n+1)(n+2) \cdots(n+k-1) k(k-1) !}{(k-1) ! k !}$

$S_{3}^{k}(n+1)=\frac{n(n+1) \cdots(n+k-1)}{k !}+\frac{(n+1)(n+2) \cdots(n+k-1) k}{k !}$

$S_{3}^{k}(n+1)=\frac{n(n+1) \cdots(n+k-1)(n+k)}{k !}$

$S_{3}^{k}(n+1)=\frac{n(n+1)(n+2) \cdots(n+1+k-1)}{k !}, n \in \square, n>0$

Para finalizar, temos que a função geradora correspondente a cada dimensão dos simplexos é dada para $\frac{x}{(1-x)^{2}}$, para os números lineares, ou seja, para $k=1$, para os números triangulares 
com $k=2$, é dada por $\frac{x}{(1-x)^{3}}$, para os tetraédricos e pentatopes, com $k=3 \mathrm{e} k=4$, respectivamente temos $\frac{x}{(1-x)^{4}}$ e $\frac{x}{(1-5)^{5}}$, então para um certo $k, k \in \square$ e $k>0$, temos a função geradora $G(x)=\frac{x}{(1-x)^{k+1}}$, de fato podemos demonstrar isto usando o produto das funções $\frac{x}{(1-x)^{k}} \cdot \frac{1}{(1-x)}$, assim temos que:

$\frac{x}{(1-x)^{k+1}}=\frac{x}{(1-x)^{k}} \cdot \frac{1}{(1-x)}=\left(S_{3}^{k-1}(1) x+S_{3}^{k-1}(2) x^{2}+\cdots+S_{3}^{k-1}(n) x^{n}\right)\left(1+x+x^{2}+\cdots x^{n-1}\right)$

$\frac{x}{(1-x)^{k+1}}=S_{3}^{k-1}(1) x+\left(S_{3}^{k-1}(1)+S_{3}^{k-1}(2)\right) x^{2}+\left(S_{3}^{k-1}(1)+S_{3}^{k-1}(2)+S_{3}^{k-1}(3)\right) x^{3}+\cdots$

$+\left(S_{3}^{k-1}(1)+S_{3}^{k-1}(2)+S_{3}^{k-1}(3)+\cdots+S_{3}^{k-1}(n)\right) x^{n}$

$\frac{x}{(1-x)^{k+1}}=S_{3}^{k}(1) x+S_{3}^{k}(2) x^{2}+S_{3}^{k}(3) x^{3}+\cdots+S_{3}^{k}(n-1) x^{n-1}+S_{3}^{k}(n) x^{n},|x|<1$

Assim, concluímos os resultados das funções geradoras dos números simplexos em dimensões arbitrárias, usando as regularidades encontradas nas relações uni, bi, tri e tetradimensionais, e artifícios algébricos que nos permitiram encontrar a generalização da classe.

\section{Conclusão}

Neste artigo foram apresentadas e discutidas, as funções geradoras de duas classes de sequência dentro dos números figurados; os números poligonais e os números simplexos ou hipertetraédricos. A escolha dessas duas classes foi baseada no crescimento tanto $\mathrm{m}$-gonal de polígonos ou seja, números triangulares, quadrados, pentagonais e assim por diante como no crescimento dimensional de politopos, que trata dos números lineares unidimensionais, números triangulares, que são os simplexos bidimensionais, os números tetraédricos, que são os análogos tridimensionais dos triângulos, ambos tendo como base os números triangulares.

Para os números poligonais, as funções geradoras tiveram um comportamento apenas no numerador, ou seja em $f(x)=a_{0}+a_{1} x+a_{2} x^{2}+\cdots+a_{n-1} x^{n-1}$, isto quer dizer que a função $g(x)=b_{0}+b_{1} x+\cdots+b_{n} x^{n}$, que é o denominador da função geradora, não se alterava. Com isso foi possível generalizar a função geradora para qualquer número poligonal em relação ao lado do polígono no qual se deseja encontrar a sequência. Por outro lado, para os números hipertetraédricos percebeu-se uma mudança apenas na função $g(x)$, sendo a função $f(x)$ definida sempre como $f(x)=1$ ou $f(x)=x$, esta última apenas para que os índices dos coeficientes da função geradora sejam os mesmos valores dos expoentes da variável, com este fato pode-se concluir uma função geradora para qualquer sequência dos análogos $k$-dimensionais dos números triangulares.

O estudo das funções geradoras para esses dois casos específicos expande a possibilidade de obtenção de outras funções dentro do estudo dos números figurados como os números de hipercubos e politopos cruzados, bem como a possibilidade de enxergar propriedade que possam ser mais complicadas quando expostas por meio da recorrência ou da fórmula do termo geral, esse escrito também traz a possibilidade de obtenção funções geradoras de qualquer sequência que possua uma recorrência linear, mesmo que não seja de números figurados por meio

BARROS, F. E.; ALVES, F. R. V. Funções geradoras dos números poligonais e dos números hipertetraédricos $k$-dimensionais. C.Q.D.- Revista Eletrônica Paulista 
da função genérica $\frac{f(x)}{g(x)}=\frac{a_{0}+a_{1} x+a_{2} x^{2}+\cdots+a_{n-1} x^{n-1}}{b_{0}+b_{1} x+b_{2} x^{2}+\cdots+b_{n} x^{n}}=c_{0}+c_{1} x+c_{2} x^{2}+\cdots+c_{n} x^{n}$. Por fim, analisar funções geradoras de sequências, bem como as estratégias algébricas usadas na obtenção destas e o estudo geométrico necessário para se trabalhar com os números figurados são parte de conteúdos importantes que podem ser explorados tantos na matemática pura quanto em situações de ensino.

\section{Referências}

ALVES, F. R. V; BORGES NETO, H.; MAIA, J. A. D. História da matemática: os números figurais em 2D e 3D. Conexões: Ciência e Tecnologia, v. 6, n. 2, p. 40-55, 2012.

ALVES, F. R. V; BARROS, F. E. Plane and space figurate numbers: visualization with the GeoGebra's help. Acta Didactica Napocensia. v. 12, n. 1, p. 57-74, 2019.

BURTON, D. M. The history of mathematics: an introduction. Boston: Allyn and Bacon, 1985.

COXETER, H. S. M. Regular polytopes. 3th ed. New York: Dover Publications, 1973.

DEZA, E; DEZA, M. M. Figurate numbers. Singapore: World Scientific, 2012.

KOSHY, T. Fibonacci and Lucas numbers with applications. New York: Wiley Interscience, 2001.

MANSOUR, T. A formula for the generating functions of powers of Horadam's sequence. Australasian Journal of Combinatorics, v. 30, p. 207-212, 2004.

VIEIRA, R. P. M.; ALVES, F. R. V. Propriedades das extensões da Sequência de Padovan. C.Q.D.- Revista Eletrônica Paulista de Matemática, v. 15, p. 24-40, jul. 2019. Edição Iniciação Científica. 